Muro de la Investigación, 2019(2), agosto-diciembre ISSN: 2523-2886

DOI: https://doi.org/10.17162/r-muro-

investigaion.v4i2.840

\title{
ESTILOS EDUCATIVOS E INTELIGENCIA EMOCIONAL EN DOCENTES DE INSTITUCIONES EDUCATIVAS CRISTIANAS DE LA CIUDAD DE JULIACA
}

\author{
Educational styles and emotional intelligence in teachers of Christian Schools \\ in the city of Juliaca
}

\author{
Santos Armandina, Farceque Huancas ${ }^{1}$ y María Elizabeth Minaya Herrera ${ }^{2 a}$ \\ Universidad Peruana Unión, Lima, Perú
}

Recibido: 12 de febrero de 2019

Aceptado: 23 de setiembre de 2019

\begin{abstract}
Resumen
El objetivo de la presente investigación fue determinar la relación que existe entre los estilos educativos y la inteligencia emocional en docentes de instituciones educativas cristianas de la ciudad de Juliaca. La metodología implica el estudio de tipo correlacional, de diseño no experimental y de corte transversal; siendo la población 150 docentes, con una muestra de 100. Para la medición de las variables se usó dos instrumentos de medición: uno sobre Perfil de estilos educativos y el Inventario de inteligencia emocional. Los resultados indican una correlación directa y significativa entre los estilos educativos y la inteligencia emocional a nivel moderado (Rho: 0.492) que viene a ser estadísticamente significativo ( $\mathrm{p}=0.006)$. Entre el estilo educativo sobreprotector y la inteligencia emocional, se halló una correlación negativa de magnitud baja $($ Rho $=-0.242)$ la cual a su vez es estadísticamente significativa $(\mathrm{p}=0.015)$. Entre el estilo educativo punitivo y la inteligencia emocional, se observa una correlación indirecta de nivel bajo $(\mathrm{Rho}=-0.334$ que es estadísticamente significativa $(\mathrm{p}=0.001)$. Entre el estilo educativo inhibicionista y la inteligencia emocional, se halló un grado de asociación muy bajo e indirecto (Rho=-0.102), sin embargo, esta no es estadísticamente significativa $(\mathrm{p}=0.314)$. En conclusión, se encontró que existe una correlación positiva entre estilos educativos y la inteligencia emocional en docentes de instituciones educativas cristianas. Asimismo, se evidenció una relación de medianamente desarrollada y estadísticamente significativa, lo que implica que, en cuanto existe mayor prevalencia de algunas de las variables como estilos educativos e inteligencia emocional, esta presenta la tendencia a generar actitudes favorables al momento de asumir la responsabilidad
\end{abstract}


de docentes y tener estrategias claras para una buena metodología con el estudiante.

Palabras clave: Estilos educativos, inteligencia emocional, instituciones educativas cristianas.

\title{
Educational styles and emotional intelligence in teachers of Christian Schools in the city of Juliaca
}

\begin{abstract}
The aim of this research was to determine the relationship between educational styles and emotional intelligence in teachers of Christian educational institutions in the city of Juliaca. The methodology involves the study of correlational type, non-experimental design and cross-sectional; the population being 150 teachers, with a sample of 100 . For the measurement of the variables two measuring instruments were used: one on Profile of educational styles and the Inventory of emotional intelligence. The results indicate a direct and significant correlation between educational styles and emotional intelligence at a moderate level (Rho: 0.492) that is statistically significant $(\mathrm{p}=0.006)$. Between the overprotective educational style and emotional intelligence, a negative correlation of low magnitude was found (Rho $=-0.242)$ which in turn is statistically significant $(\mathrm{p}=0.015)$. Between the punitive educational style and emotional intelligence, an indirect correlation of low level is observed ( $R$ ho $=-0.334$ which is statistically significant $(p=0.001)$. Between the inhibitionist educational style and emotional intelligence, a very high degree of association was found low and indirect (Rho $=-0.102$ ), however, this is not statistically significant $(\mathrm{p}=0.314)$ In conclusion, it was found that there is a positive correlation between educational styles and emotional intelligence in teachers of Christian educational institutions. a relationship of moderately developed and statistically significant was evidenced, which implies that, as there is a higher prevalence of some of the variables such as educational styles and emotional intelligence, this presents the tendency to generate favorable attitudes when assuming the responsibility of teachers and have clear strategies for a good methodology with the student.
\end{abstract}

Keywords: Educational styles, emotional intelligence, Christian educational institutions.

\section{Introducción}

Según la Organización de las Naciones Unidas para la Educación, la Ciencia y la Cultura (UNESCO, 2018), la salud mental del docente en América Latina se encuentra afectada principalmente por el estrés y la depresión; siendo el estrés el mayor porcentaje de incidencia en los docentes. Por ejemplo, en Argentina, el 34\% de docentes encuestados, resultó con un tipo grave de estrés; en Chile, fue el 42\%; en Ecuador, el 48\%; en México, el 27\%; en Uruguay, el 34\% y en el Perú el 37\%. Las causas de este cuadro con frecuencia 
tienen que ver con el poco tiempo de descanso durante la jornada laboral, el trabajo realizado fuera de las horas académicas de labor asignadas, el poco apoyo de los padres de familia, las condiciones sociales del alumnado y las exigencias de las autoridades relativas a la educación y la formación académica (UNESCO, 2015).

Por otro lado, la inteligencia emocional se plantea como el conjunto de emociones y destrezas que influyen en la habilidad para adaptarse y enfrentar las demandas y presiones del medio. La inteligencia no cognitiva es un factor importante en la determinación de las habilidades para tener éxito en la vida, influyendo directamente en el bienestar general y en la salud emocional. El resultado de ello nos conlleva a desarrollar buenos estilos educativos, que vienen a ser el conjunto de ideas, creencias, valores, actitudes y hábitos de comportamiento-que especialmente padres y docentes mantienen respecto a la educación de sus hijos y los estudiantes (Bar-On, citado por Ugarriza, 2001).

Según Magaz y García (1998) los estilos educativos vienen a ser el conjunto de ideas, creencias, valores, actitudes y hábitos de comportamiento que especialmente padres y docentes mantienen respecto a la educación de los hijos y estudiantes. Asimismo, los mismos autores afirman que todas las personas, al estar inmersas en un medio social, son influenciadas por el entorno desde la infancia hasta la madurez, tanto a través de modelos directos (padres, hermanos, tíos, abuelos y profesores) como de modelos indirectos (la televisión, prensa, radio, etc.). De igual manera, es muy importante que las personas desarrollen la inteligencia emocional, a fin que faciliten la interacción con las personas y logren adquirir un adecuado bienestar emocional. Por ende, la inteligencia emocional se refiere a la capacidad humana de sentir, entender, controlar y modificar estados emocionales en uno mismo y en los demás (Bar-On, 1997).

Respecto a la correlación entre la variable "estilo educativo asertivo" y la inteligencia emocional se observa una correlación directa a nivel moderado, que viene a ser estadísticamente significativa. Esto quiere decir que, a mayor capacidad de manejo y regulación de emociones tanto a nivel interpersonal, intrapersonal, manejo de estrés, adaptabilidad y estado de ánimo general, existe la probabilidad de que el docente utilice el estilo asertivo como estrategia de enseñanza y formación pedagógica. Este resultado, según Bar-On (citado por Ugarriza, 2001), evidenciarían un comportamiento equilibrado, capaz de afrontar las demandas del entorno, tal y como Extremera, Fernández-Berrocal y 
Durán (2003) concluyen tras encontrar que los docentes que poseen ciertas habilidades emocionales pueden hacerles frente a niveles intensos de estrés y ansiedad y evitar desajustes emocionales serios. Esto no se observaría en un docente con estilo sobreprotector, ya que según Magaz y García (1998), el estilo sobreprotector evidencia un fuerte e intenso sentimiento de responsabilidad, así como la culpabilización frente a los errores que sus estudiantes pudieran cometer. Por su parte, Villán (2013) encontró que los docentes con inteligencia emocional desarrollada, evidencian mejor interacción con sus estudiantes como liderazgo afectivo y resolución de conflictos. En cuanto al estilo asertivo, se observa relación significativa y, como refiere Sala (2002), esto puede deberse a la influencia positiva de la inteligencia emocional en los estilos educativos.

Este panorama conlleva a formular los objetivos de determinar la relación que existe entre los estilos educativos y la inteligencia emocional en docentes de instituciones educativas cristianas de la ciudad de Juliaca. Por ende, el objetivo específico de esta investigación es: determinar la relación que existe entre el estilo educativo asertivo, sobreprotector, punitivo e inhibicionista en docentes de instituciones educativas cristianas de la ciudad de Juliaca.

\section{Método}

Diseño

La presente investigación se enmarca en un tipo correlacional, pues tiene el objetivo de asociar dos o más variables bajo un patrón predecible (Hernández, Fernández y Baptista, 2014). En este contexto, se propone vincular los estilos educativos y sus dimensiones con la variable "inteligencia emocional", buscando de esta forma correlacionar y encontrar respaldo estadístico y correlación entre la variable predictora y la variable criterio en docentes de instituciones adventistas. Debido a que en la presente investigación no se realizó alguna manipulación de las variables de estudio, sino que estas fueron observadas en su ambiente natural, es que corresponde a un diseño no experimental de corte transversal transeccional, pues la información se recolecto en un solo momento en la línea del tiempo (Hernández, Fernández y Baptista, 2014).

Participantes 
La población de estudio estuvo conformada por 150 docentes activos en instituciones educativas cristianas de la ciudad de Juliaca. Estas fueron: El Buen Pastor, Pedro Kalbermater, Luz y Ciencia, Fernando Stahl, Andrés Bello y Eduardo Forga. La edad de los docentes oscila entre los 20 a 57 años. El tipo de muestreo fue no probabilístico, a conveniencia del investigador. Debido a que la población no es alcanzable en su totalidad se trabajó con una muestra representativa de 100, halladas mediante la fórmula de muestreo para poblaciones finitas, asimismo, estos fueron elegidos mediante una técnica probabilística correspondiente al tipo aleatorio simple.

\section{Instrumentos}

A su vez, se utilizaron los siguientes instrumentos: el Perfil de Estilos Educativos (PEE), elaborado y validado por Magaz y García (1998) con un alfa de Cronbach de 0.79; este consta de 48 afirmaciones con alternativa de respuesta dicotómica. Dicho perfil pretende valorar cualitativa y cuantitativamente las actitudes y valores ante la educación de los alumnos e hijos, así también permite cuantificar las ideas, actitudes y valores correspondientes a los estilos sobreprotector, punitivo, inhibicionista y asertivo. El otro instrumento es el Inventario de inteligencia Bar-On, creado por Reuven Bar-On (2004); traducido y adaptado para uso experimental de la investigación, en el Perú por: Zoila Abanto, Leonardo Higueras, y Jorge Cueto, su administración es individual colectiva, con un tiempo de 20 - 50 minutos, y es aplicable desde los 16 años en adelante. Esta prueba contiene133 ítems, compuesto de cinco dimensiones: relaciones intrapersonales, relaciones interpersonales, adaptabilidad, manejo del estrés y finalmente el estado de ánimo general. Estos deben ser respondidos a través de una escala tipo Likert de 1 a 5 , desde nunca, hasta siempre (Román Murillo, 2016).

\section{Resultados}

Se obtuvo el análisis descriptivo de las variables de estudio, inteligencia emocional, y se observa que el 98\% de encuestados evidencian un nivel de inteligencia emocional medianamente desarrollada mientras que solo un $2 \%$ necesita mejorar. Por otra parte, en cuanto a los estilos educativos, se observa que el $73 \%$ de los encuestados evidencia una 
predominancia alta de estilo asertivo, un $70 \%$ evidencia un nivel moderado de estilo sobreprotector, un $60 \%$ un nivel moderado de estilo punitivo y un $74 \%$ un nivel moderado de estilo inhibicionista. Más bien, en cuanto a los porcentajes bajos se evidencia que un $23 \%$ presume un nivel moderado de estilo asertivo, así también, un 17\% un nivel bajo de estilo sobreprotector, un $33 \%$ un nivel bajo de estilo punitivo y un $20 \%$ un nivel bajo de estilo inhibicionista.

Tabla 1

Niveles de inteligencia emocional en la población encuestada

$\begin{array}{llcc} & & \text { Frecuencia } & \text { Porcentaje } \\ \text { Inteligencia emocional } & \text { Necesita mejorar } & 2 & 2 . \% \\ & \text { Medianamente desarrollado } & 98 & 98 . \% \\ & \text { Total } & 100 & 100 . \% \\ \text { Estilo asertivo } & \text { Bajo } & 4 & 4 . \% \\ & \text { Moderado } & 23 & 23 . \% \\ & \text { Alto } & 73 & 73 . \% \\ \text { Estilo sobreprotector } & \text { Total } & 100 & 100 . \% \\ & \text { Bajo } & 17 & 17 . \% \\ & \text { Moderado } & 70 & 70 . \% \\ & \text { Alto } & 13 & 13 . \% \\ & \text { Total } & 100 & 100 . \% \\ & \text { Bajo } & 33 & 33 . \% \\ & \text { Moderado } & 60 & 60 . \% \\ & \text { Alto } & 7 & 7 . \% \\ & \text { Total } & 100 & 100 . \% \\ & \text { Bajo } & 20 & 20 . \% \\ & \text { Moderado } & 74 & 74 . \% \\ & \text { Alto } & 6 & 6 . \% \\ & \text { Total } & 100 & 100 . \%\end{array}$

Según el análisis de normalidad de las variables, se observan valores inferiores al .05 como resultado de la prueba de normalidad en la variable estilo asertivo, sobreprotector, punitivo e inhibicionista: mientras que un valor por encima del .05 en la variable "inteligencia emocional." Por ello, ante la evidente superioridad de valores inferiores al .05, se asume aceptar la hipótesis alterna, la cual refiere que los datos no provienen de una distribución normal. Por lo tanto, el análisis estadístico adecuado correspondería a uno no paramétrico, para este caso, Rho de Spearman o Tau B de Kendall, 
como coeficientes de correlación para la prueba de hipótesis.

Tabla 2

Prueba de normalidad según Kolmogorov smirnov para una muestra

\begin{tabular}{|c|c|c|c|c|c|c|}
\hline & \multirow{2}{*}{$\begin{array}{r}\begin{array}{r}\text { Inteligencia } \\
\text { emocional }\end{array} \\
100\end{array}$} & \multirow{2}{*}{$\begin{array}{r}\text { Asertivo } \\
100\end{array}$} & \multirow{2}{*}{$\begin{array}{r}\text { Sobreprotector } \\
100\end{array}$} & \multirow{2}{*}{$\begin{array}{r}\text { Punitivo } \\
100\end{array}$} & \multirow{2}{*}{$\begin{array}{r}\text { Inhibicionista } \\
100\end{array}$} \\
\hline $\mathrm{N}$ & & & & & & \\
\hline Parámetros & Media & $\begin{array}{l}379,090 \\
0\end{array}$ & 21,4600 & 17,8700 & 16,7200 & 17,3000 \\
\hline \multirow[t]{2}{*}{ normales $^{\mathrm{a}, \mathrm{b}}$} & Desviación & $\begin{array}{l}41,6883 \\
8\end{array}$ & 2,48397 & 2,47678 & 2,39562 & 2,14382 \\
\hline & estándar & & & & & \\
\hline Máximas & Absoluta & ,069 & ,246 & ,114 & , 188 & ,116 \\
\hline diferencias & Positivo & 055 & ,143 & ,114 & ,188 & ,116 \\
\hline extremas & Negativo &,- 069 &,- 246 &,- 091 &,- 108 &,- 104 \\
\hline \multicolumn{2}{|c|}{ Estadístico de prueba } & 069 & 246 &, 114 & , 188 & ,116 \\
\hline \multicolumn{2}{|c|}{ Sig. asintótica (bilateral) } &, $200^{\mathrm{c}, \mathrm{d}}$ &, $000^{c}$ &, $003^{c}$ &, $000^{\mathrm{c}}$ &, $002^{\mathrm{c}}$ \\
\hline \multicolumn{7}{|c|}{$\begin{array}{l}\text { a. La distribución de prueba es } \\
\text { normal. }\end{array}$} \\
\hline \multicolumn{7}{|c|}{ b. Se calcula a partir de datos. } \\
\hline \multicolumn{2}{|c|}{$\begin{array}{l}\text { c. Corrección de significación de } \\
\text { Lilliefors. }\end{array}$} & & & & & \\
\hline
\end{tabular}

Asimismo, se realizó el análisis diferencial mediante una prueba no paramétrica, puesto que los datos no provienen de una distribución normal. En ese sentido, según la tabla 7 respecto a la correlación entre la variable estilo educativo asertivo en su escala total y la inteligencia emocional, se observa una correlación directa a nivel moderado (Rho $=.492$ ), que viene a ser estadísticamente significativa $(\mathrm{p}=.006)$. Esto quiere decir que, a mayor capacidad de manejo y regulación de emociones tanto a nivel interpersonal, intrapersonal, manejo de estrés, adaptabilidad y estado de ánimo general, existe la probabilidad de que el docente utilice el estilo asertivo como estrategia de enseñanza y formación pedagógica.

En cuanto a la correlación entre la variable "estilo educativo sobreprotector" y "la inteligencia emocional," se halló una correlación negativa de magnitud baja (Rho = -.242) la cual a su vez es estadísticamente significativa $(\mathrm{p}=.015)$. Esto indica que, a menor capacidad de manejo y regulación de emociones en el docente, existe la probabilidad certera de que predomine en él un estilo educativo sobreprotector; es decir, el educador piensa que 
el niño es un ser débil, ignorante, inexperto, a quien hay que proteger, evitando que se exponga a situaciones de riesgo en las que pueda sufrir algún perjuicio.

En cuanto a la correlación entre la variable "estilo educativo punitivo" y la inteligencia emocional, se observa una correlación indirecta de nivel bajo (Rho = -.334) que a la vez es estadísticamente significativa $(\mathrm{p}=.001)$. Esto indica que, a menor capacidad de manejo y regulación de las emociones por parte de los docentes existe una probabilidad certera de que predomine en ellos un estilo educativo basado en no permitir ningún fallo o desviación del comportamiento normativo, además de no reconocer el esfuerzo para adquirir un hábito o una habilidad, durante lo cual se presentarán fallos, errores, equivocaciones, insuficiencias, todo esto por parte del estudiante.

Finalmente, respecto a la correlación entre la variable estilo educativo inhibicionista y la inteligencia emocional se halló un grado de asociación muy bajo e indirecto (Rho = .102). Sin embargo, esta no es estadísticamente significativa ( $\mathrm{p}=.314$ ); por lo tanto, cualquier aseveración respecto a estas dos variables no tendría asidero metodológico pues este resultado ha sobrepasado el margen de error.

\section{Tabla 3}

Análisis de correlación entre la variable inteligencia emocional y las dimensiones de la variable estilos educativos

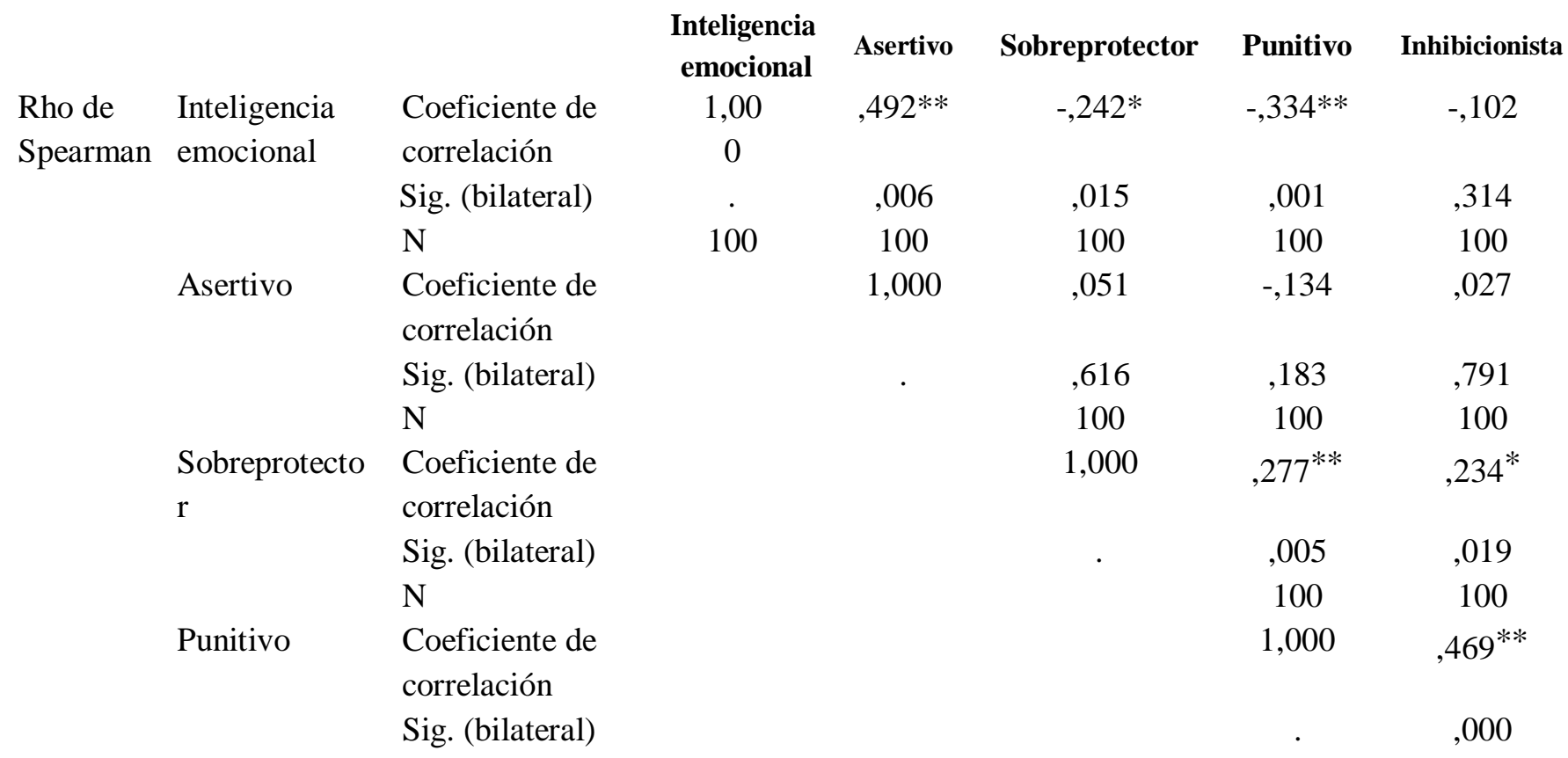


correlación

Sig. (bilateral)

$\mathrm{N}$

*. La correlación es significativa en el nivel

0,05 (bilateral).

**. La correlación es significativa en el nivel

0,01 (bilateral).

\section{Discusión}

El objetivo de la presente investigación estuvo enfocado en determinar si existe relación significativa entre los estilos educativos y la inteligencia emocional en docentes de colegios adventistas de la ciudad de Juliaca, que no integran la asociación educativa adventista Puno - 2018. Respecto a la correlación entre la variable "estilo educativo asertivo" y la inteligencia emocional, se observa una correlación directa a nivel moderado que viene a ser estadísticamente significativa: a mayor capacidad de manejo y regulación de emociones tanto a nivel interpersonal, intrapersonal, manejo de estrés, adaptabilidad y estado de ánimo general, existe la probabilidad de que el docente utilice el estilo asertivo como estrategia de enseñanza y formación pedagógica. Este resultado, donde la inteligencia emocional se encuentra en niveles adecuados o muy desarrollados, evidenciarían un comportamiento equilibrado, capaz de afrontar las demandas del entorno, tal y como Extremera, Fernández-Berrocal y Durán (2003) concluyen tras encontrar que los docentes que poseen ciertas habilidades emocionales pueden hacerles frente a niveles intensos de estrés y ansiedad y evitar desajustes emocionales serios. Esto no se observaría en un docente con estilo sobreprotector, ya que según Magaz y García (1998), el estilo sobreprotector evidencia un fuerte e intenso sentimiento de responsabilidad, así como la culpabilización frente a los errores que sus estudiantes pudieran cometer. Villán (2013) también encontró que docentes con inteligencia emocional desarrollada, evidencian mejor interacción con sus estudiantes como liderazgo afectivo y resolución de conflictos. En cuanto al estilo asertivo se observa relación significativa y como refiere Sala (2002), esto puede deberse a que la influencia de la inteligencia emocional en los estilos educativos es desarrollada. 
En cuanto a la correlación entre la variable "estilo educativo sobreprotector" y la inteligencia emocional se halló una correlación negativa de magnitud baja, la cual es estadísticamente significativa. Esto indica que, a menor capacidad de manejo y regulación de emociones en el docente, existe la probabilidad certera de que predomine en él un estilo educativo sobreprotector; es decir, el educador piensa que el niño es un ser débil, ignorante, inexperto, a quien hay que proteger, evitando que se exponga a situaciones de riesgo en las que pueda sufrir algún perjuicio. Esto lo corroboran Sala (2002) y Dorta (2012), quienes refieren que una mayor puntuación en el estilo sobreprotector se correspondía con menores habilidades de inteligencia emocional.

Por otro lado, en la población de docentes de nivel secundario, se encontró una correlación negativa entre las variables de estudio, concluyendo que ciertas habilidades emocionales que los docentes poseen sirven como protectores en los desajustes emocionales (según Extremara, Fernández- Berrocal y Duran, 2003). Cabe resaltar que las teorías de la inteligencia emocional de Daniel Goleman (1995) y las inteligencias múltiples de Howard Gardner (2011), que predominan en el estudio de la psicología y la educación, enfatizan que la inteligencia abarca mayores destrezas y capacidades del individuo, desarrollándose en una que va más allá de habilidades ligadas a la lógica, en la que cada uno nos valemos de diferentes recursos para alcanzar el éxito en la vida.

En cuanto a la correlación entre la variable "estilo educativo punitivo" y la inteligencia emocional, se observa una correlación indirecta de nivel bajo, la cual a su vez es estadísticamente significativa: a menor capacidad de manejo y regulación de las emociones por parte de los docentes existe una probabilidad certera de que predomine en ellos un estilo educativo basado en no permitir ningún fallo o desviación del comportamiento normativo, además de no reconocer el esfuerzo para adquirir un hábito o una habilidad. Esto define la teoría de Bar-On (1997), que el manejo del estrés consta de la habilidad para tolerar y soportar situaciones adversas enfrentándolas de manera activa y positiva, así como la capacidad para postergar un impulso. Mientras que Magáz y García (1998) refieren que el estilo punitivo está caracterizado por la intolerancia e incomprensión, así como una falta de control de impulsos. Ante esto, Dorta (2012) encontró que bajas puntuaciones en el conocimiento de las propias emociones se correlacionó con padres con tendencia al perfeccionismo y trato hostil, padres 
censuradores centrados en la conducta negativa de sus hijos.

Finalmente, respecto a la correlación entre la variable "estilo educativo inhibicionista" y la inteligencia emocional, se halló un grado de asociación muy bajo e indirecto; sin embargo, esta no es estadísticamente significativa. Por lo tanto, cualquier aseveración respecto a estas dos variables no tendría asidero metodológico pues este resultado ha sobrepasado el margen de error. Como refiere Sala (2002), esto puede deberse a que la influencia de la inteligencia emocional en los estilos educativos es moderada y compleja, dándose la probabilidad de que incidan otros factores. Asimismo, los estilos educativos del profesor pueden influir en el desarrollo emocional y social del alumno, por lo que se comprende que las prácticas educativas están estrechamente relacionadas con la socialización emocional del mismo. En este aspecto, Parke et al (1989) señalan que la familia educa las conductas emocionales de los niños a partir de influencias directas o indirectas en el curso de la socialización emocional.

\section{Referencias}

Ba-On, R. (1997). The Emotional Quotient Inventory (EQ-i): A test of emotional intelligence. Toronto: Multi-Health Systems.

Extremera, N., Fernández-Berrocal, P., Durán, A. (2003). Inteligencia emocional y burnout en profesores. (Trabajo de investigación, Universidad de Málaga España). Recuperado de http://emotional.intelligence.uma.es/documentos/PDF5burnout_en_profesor es.pdf.

Extremera, N. y Fernández-Berrocal, P. (2004). La importancia de desarrollar la inteligencia emocional en el profesorado. Revista Iberoamericana de Educación. Recuperado de http://www.rieoei.org/deloslectores/759Extremera.PDF

García-Fernández, M., Giménez-Mas, S. (2010). La inteligencia emocional y sus principales modelos: propuesta de un modelo integrador. Espiral. Cuadernos del Profesorado, 3(6), 43-52. Recuperado de http://www.cepcuevasolula.es/espiral/articulos/ESPIRAL_VOL_3_N_6_ART_4.pdf

Gardner, H. (1993). Inteligencias múltiples: la teoría en la práctica. Barcelona: Paidos. Goleman, D. (1999). La práctica de la inteligencia emocional. New York. España: Editorial Kairós S.A. 
Hernández R., Fernández C. y Baptista M. (2010) Metodología de la Investigación Quinta edición. D.F. - México. Interamericana Editores, S.A.

Magaz, Á. y García, M. (1998). Perfil de estilos educativos. Manual de referencia. España: ALBOR-COHS.

Sala, J., \& Abarca, M. (2002). Las competencias emocionales de los futuros profesores. Revista Electrónica Interuniversitaria de Formación del Profesorado, 5(3), 1-4. Retrieved from https://dialnet.unirioja.es/servlet/articulo?codigo=1034544

Segura Yauri, A. S. (2016). Inteligencia emocional y estilos educativos en docentes de Instituciones Privadas Religiosas de Chosica, 2015. Universidad Peruana Unión. Retrieved from http://repositorio.upeu.edu.pe/handle/UPEU/467.

Ugarriza, N. \& Pajares, L. (2001). Adaptación y estandarización del Inventario de Inteligencia Emocional de BarOn ICE: NA, en niños y adolescentes. Manual técnico. Lima: Ediciones La Aurora. 